\title{
A comprehensive overview and qualitative analysis of government-led nutrition policies in Australian institutions
}

Emalie Rosewarne ${ }^{1 *}$ D, Annet C. Hoek ${ }^{1}$, Gary Sacks ${ }^{2}$, Luke Wolfenden ${ }^{3}$, Jason Wu', Jenny Reimers ${ }^{4}$, Kirstan Corben ${ }^{4}$, Michael Moore ${ }^{1}$, Cliona Ni Mhurchu ${ }^{1,5}$ and Jacqui Webster ${ }^{1}$

\begin{abstract}
Background: Institutions are a recommended setting for dietary interventions and nutrition policies as these provide an opportunity to improve health by creating healthy food environments. In Australia, state and territory governments encourage or mandate institutions in their jurisdiction to adopt nutrition policies. However, no work has analysed the policy design across settings and jurisdictions. This study aimed to compare the design and components of government-led institutional nutrition policies between Australian states and territories, determine gaps in existing policies, and assess the potential for developing stronger, more comprehensive policies.

Methods: Government-led institutional nutrition policies, in schools, workplaces, health facilities and other public settings, were identified by searching health and education department websites for each Australian state and territory government. This was supplemented by data from other relevant stakeholder websites and from the Food Policy Index Australia website. A framework for monitoring and evaluating nutrition policies in publicly-funded institutions was used to extract data and a qualitative analysis of the design and content of institutional nutrition policies was performed. Comparative analyses between the jurisdictions and institution types were conducted, and policies were assessed for comprehensiveness.

Results: Twenty-seven institutional nutrition policies were identified across eight states and territories in Australia. Most policies in health facilities and public schools were mandatory, though most workplace policies were voluntary. Twenty-four included nutrient criteria, and 22 included guidelines for catering/fundraising/advertising. While most included implementation guides or tools and additional supporting resources, less than half included tools/timelines for monitoring and evaluation. The policy design, components and nutrient criteria varied between jurisdictions and institution types, though all were based on the Australian Dietary Guidelines.

Conclusions: Nutrition policies in institutions present an opportunity to create healthy eating environments and improve population health in Australia. However, the design of these policies, including lack of key components such as accountability mechanisms, and jurisdictional differences, may be a barrier to implementation and prevent the policies having their intended impact.
\end{abstract}

Keywords: Food policy, Nutrition standards, Institutions, Qualitative research, Australia

\footnotetext{
* Correspondence: erosewarne@georgeinstitute.org.au

'The George Institute for Global Health, The University of New South Wales, Sydney, NSW 2052, Australia

Full list of author information is available at the end of the article
}

(c) The Author(s). 2020 Open Access This article is licensed under a Creative Commons Attribution 4.0 International License, which permits use, sharing, adaptation, distribution and reproduction in any medium or format, as long as you give appropriate credit to the original author(s) and the source, provide a link to the Creative Commons licence, and indicate if changes were made. The images or other third party material in this article are included in the article's Creative Commons licence, unless indicated otherwise in a credit line to the material. If material is not included in the article's Creative Commons licence and your intended use is not permitted by statutory regulation or exceeds the permitted use, you will need to obtain permission directly from the copyright holder. To view a copy of this licence, visit http://creativecommons.org/licenses/by/4.0/ The Creative Commons Public Domain Dedication waiver (http://creativecommons.org/publicdomain/zero/1.0/) applies to the data made available in this article, unless otherwise stated in a credit line to the data. 


\section{Background}

In Australia, dietary risks are the second leading mortality risk factor, and responsible for an estimated $14.8 \%$ of total deaths [1]. Key dietary risk factors include low intakes of wholegrains, fruit, nuts and seeds, vegetables, fibre, omega-3 fatty acids, polyunsaturated fatty acids and legumes, and excessive sodium consumption, which are all estimated to be among the top 20 mortality risk factors [1]. Many strategies have been initiated, both in Australia and globally, to address the disease burden attributable to dietary risks. In Australia, these strategies include government-led voluntary nutrient reformulation targets [2, 3], education and awareness raising campaigns (e.g. [4]), interpretive front-of-pack labelling [5], and institutional nutrition policies (e.g. [6]).

Institutions, including schools, workplaces and health facilities, are a recommended setting for dietary interventions $[7,8]$, as they provide access to a large proportion of the population for a prolonged period of time [9, 10] and have existing infrastructure to deliver interventions. One strategy to influence dietary intake, and achieve improvements in population health, is by setting standards or guidelines on the foods and drinks an institution can procure, prepare, provide and sell. Such nutrition policies are particularly important in public institutions, where governments have the ability to define these standards or guidelines, and encourage or mandate adoption of the policies, and can set an example for creating healthy eating environments [7, 8]. Previous studies suggest that these nutrition policies and guidelines, can be effective in increasing availability and purchases of healthy foods in many environments, including institutions [11]. However, the evidence is limited to high-income countries and few interventions have been subject to rigorous evaluations [11]. In addition, compared to the number of known institutional nutrition policies in existence [12], a relatively small proportion have been evaluated, highlighting a key area for future work.

Institutional nutrition policies and guidelines can be implemented at various levels of government, including national, state/provincial or local governments, depending on each level's jurisdiction [13]. In Australian institutions, nutrition policies and guidelines exist at multiple levels of government. There are some voluntary national policies and guidelines (e.g. [6]), however regulations pertaining to many institutions, for example schools and hospitals, are the responsibility of the state and territory governments [14]. As such, many jurisdictions have developed their own policies (e.g. $[15,16])$, and state/territory governments encourage or mandate institutions in their jurisdiction to adopt these policies.

It is important to understand whether these state and territory level institutional nutrition policies are consistent with evidence based recommendations (e.g. [13]), to what extent they are being implemented, and the effect of the policies on the food environment, dietary intake or downstream health outcomes. One of the challenges is that the available evidence is limited to particular institutions and settings. The majority of previous research on government-led institutional nutrition policies in Australia has centred on schools [17], with minimal research relating to health facilities and none relating to workplace nutrition policies. Previous work regarding schools has included investigations into compliance with policies and stakeholder perceptions $[18,19]$, an overview of differences in policy design and implementation across states and territories [20], and a few randomised control trials in the Hunter region of New South Wales [21-23]. However, since these studies were conducted, the Australian Capital Territory [15], New South Wales [24], Northern Territory [25] and Western Australia [26] policies have been revised, and an update of this research would therefore be warranted. In contrast, little research has been conducted on nutrition policies in health facilities. Studies have been limited to assessing compliance in a small number of health facilities, through audits of foods and drinks available [27, 28].

It is evident that the focus of past research was on policy compliance, yet no work has analysed the policy design, including the extent to which they are consistent with evidence-based recommendations (e.g. [13]), or assessed the potential for developing more comprehensive nutrition policies for Australian institutions. Therefore, the aim of this study was to compare the design and components of government-led institutional nutrition policies between Australian states and territories, determine gaps in existing policies, and assess the potential for developing stronger, more comprehensive policies. Implementation of the policies, and the impact of the policies on total dietary intake or downstream health outcomes is also yet to be assessed, however this is outside the scope of the current paper.

\section{Methods}

Health and education department websites for each state and territory government were searched to identify current government-led nutrition policies relating to institutions, including schools, workplaces, health facilities, or other public settings. From the state and territory government websites, other relevant stakeholder websites, including federal government and quasi-government, were identified and searched. Information from the Food Policy Index Australia website, a website that summarises the progress made by Australian governments in line with implementing globally recommended policies, was used to supplement these data [29]. 


\section{Inclusion and exclusion criteria}

For this study, nutrition policies were defined as policies, guidelines or frameworks pertaining to creating healthy eating and drinking environments within the institution (i.e. increasing the availability and purchases of healthy foods $[11,13])$, such as through nutrition standards. Policies for the provision of food to institutionalised individuals (e.g. hospital in-patients) were excluded as these do not aim to improve the food environment, and food for these populations is wholly provided by the government, requiring $100 \%$ of daily nutrient needs to be met.

\section{Data extraction and analysis}

To compare the design and components of governmentled institutional nutrition policies, determine gaps in existing policies, and assess the potential for developing stronger, more comprehensive policies, three levels of comparative policy analysis were undertaken: (1) Policy design and components, (2) nutrition standards, and (3) nutrient criteria. Relevant data were extracted from the policy documents by one author (E.R.) and entered into a purpose-built Microsoft Excel spreadsheet based on L'Abbé et al.'s framework for monitoring and evaluating nutrition standards in publicly-funded institutions [13]. For the comparative policy analysis, institution type, policy name and year introduced/revised were extracted, as well as policy design elements such as approach (voluntary/mandatory), components of the policy (nutrition standards, voluntary policy template, implementation guide/tools, guide for catering/fundraising/advertising, extra resources to support implementation, monitoring/ evaluation guide/tools, accreditation program), outline of assigned roles and responsibilities, and outline of monitoring and evaluation plan (including qualitative and/or quantitative approach and time points). Data extracted relating to nutrition standards included: policy name, nutrient profiling system used (e.g. traffic light labelling/ Health Star Rating (HSR, [5])), and frequency of specific foods permitted in food outlets/canteens and for catering, fundraising and advertising. Information on nutrient criteria was extracted from the nutrition standards documents and compared between jurisdictions for schools, workplaces and health facilities. Nutrient criteria included guidelines or thresholds relating to energy, saturated fat, sodium, sugar and fibre contents (per $100 \mathrm{~g}$ and per serve), portion size criteria and Health Star Rating cut-offs where applicable [5].

\section{Results}

\section{Comparison of policy design and components}

Twenty-seven government-led state and territory level institutional nutrition policies were identified across all eight jurisdictions, including seven policies relating to health facilities, eight school nutrition policies, eight workplace policies, and four policies in other settings. Policies varied between states/territories and between types of institutions. Most policies in health facilities and public schools were mandatory, as was the Australian Capital Territory public sector policy, which applies to all public sector workplaces and facilities. All other workplace policies and all policies in other settings were voluntary, as was the Tasmanian schools policy and Victorian health facilities policies. The components of the policies also varied. Of the 27 policies, 24 included nutrition standards (of which 16 were mandatory and eight were voluntary), 22 included guidelines for catering/fundraising/advertising, 21 included implementation guides or tools, 12 included monitoring/evaluation resources, eight provided a voluntary policy template for modification, seven had an accreditation program available and 25 included additional supporting resources. Six out of seven health facility policies and six out of eight school policies outlined roles and responsibilities for implementation and monitoring, though only one workplace policy included this. The inclusion of plans for ongoing monitoring and evaluation was variable, with four out of seven health facility policies and two out of eight school policies specifying evaluation/review timelines, and two out of eight workplace policies recommending timelines. Four workplace and three school policies had accreditation programs. No state or territory had an accreditation program explicitly for health facility policies, however Victorian health services are encouraged to use the workplace program (Table 1).

\section{Comparison of nutrition standards}

Most schools and health facilities with nutrition policies had a mandatory nutrition standards, as did the Australian Capital Territory public sector policy. Four state/territory workplace policies, three policies in other settings, one school and one health facility policy included voluntary nutrition standards. All nutrition standards were based on the Australian Dietary Guidelines [138] classification of core and discretionary foods, however two different systems were used to classify the foods. Except for New South Wales, all other states and territories utilised a traffic light labelling system (Table 2). Traffic light classifications were specified as: Red (described as 'not recommended', 'not on the menu'/'off the menu', 'occasional' foods or foods to 'avoid'/'limit'), amber ('select carefully'/'choose carefully') and green (described as the 'best choice', 'always on the menu'/'fill the menu', 'have plenty', or 'everyday' foods). However, the state of New South Wales used the HSR system and portion size criteria to classify foods into 'everyday' and 'occasional' foods, where 'everyday' foods were described as foods from the five food groups and 'occasional' foods as foods with less nutritional value than everyday foods [24]. 
Table 1 Comparison of the design and components of state and territory nutrition policies in Australian institutions

\begin{tabular}{|c|c|c|c|c|c|}
\hline $\begin{array}{l}\text { State/territory and year } \\
\text { introduced or most } \\
\text { recently updated }\end{array}$ & Name of policy/guideline & Approach & Components & $\begin{array}{l}\text { Outline } \\
\text { of roles/ } \\
\text { responsibilities }\end{array}$ & $\begin{array}{l}\text { Outline of monitoring } \\
\text { and evaluation plan }\end{array}$ \\
\hline \multicolumn{6}{|l|}{ Health facilities } \\
\hline $\begin{array}{l}\text { Australian Capital } \\
\text { Territory; } 2018\end{array}$ & $\begin{array}{l}\text { Healthy Food and Drink Choices } \\
\text { Policy }[16,30-32]\end{array}$ & Mandatory & $N S, G, R, M / E$ & Yes & $\begin{array}{l}12 \text { months, } 24 \text { months } \\
\text { only (qualitative and } \\
\text { quantitative) }\end{array}$ \\
\hline New South Wales; 2017 & $\begin{array}{l}\text { Healthy Food and Drink in NSW } \\
\text { Health Facilities for Staff and } \\
\text { Visitors Framework }[33,34]\end{array}$ & Mandatory & $N S, I, G, M / E$ & Yes & Annual (quantitative) \\
\hline Northern Territory; 2017 & $\begin{array}{l}\text { Healthy Choices Made Easy - } \\
\text { Healthy Food and Drink Options } \\
\text { for Staff, Volunteers and Visitors in } \\
\text { NT Heath Facilities Policy [35-40] }\end{array}$ & Mandatory & $N S, G, R$ & No & $\begin{array}{l}\text { Baseline, } 6 \text { months, } 18 \\
\text { months only (qualitative } \\
\text { and quantitative) }\end{array}$ \\
\hline $\begin{array}{l}\text { Queensland; } 2015 \\
\text { (currently being revised) }\end{array}$ & $\begin{array}{l}\text { A Better Choice. Healthy Food } \\
\text { and Drink Supply [41-45] }\end{array}$ & Mandatory & $N S, I, G$ & Yes & No \\
\hline $\begin{array}{l}\text { South Australia; } 2011 \\
\text { (review planned) }\end{array}$ & $\begin{array}{l}\text { Healthy Food and Drink Choices } \\
\text { for Staff and Visitors in SA Health } \\
\text { Facilities [46-53] }\end{array}$ & Mandatory & $N S, I, G, R$ & Yes & No \\
\hline Tasmania & Nil & N/A & N/A & N/A & N/A \\
\hline Victoria; 2016 & $\begin{array}{l}\text { Healthy choices: policy guidelines } \\
\text { for hospitals and health } \\
\text { services }[54,55]\end{array}$ & Voluntary & $N S, I, G, R, M / E$ & Yes & $\begin{array}{l}6 \text { months, then } \\
\text { annually (qualitative } \\
\text { and quantitative) }\end{array}$ \\
\hline Western Australia; 2017 & $\begin{array}{l}\text { The Healthy Options WA: Food } \\
\text { and Nutrition Policy for WA Health } \\
\text { Services and Facilities [56-61] }\end{array}$ & Mandatory & $N S, I, G, R, M / E$ & Yes & No \\
\hline \multicolumn{6}{|l|}{ Schools } \\
\hline $\begin{array}{l}\text { Australian Capital } \\
\text { Territory; } 2017\end{array}$ & $\begin{array}{l}\text { ACT Public School Food and Drink } \\
\text { Policy [15, 62-70] / National Healthy } \\
\text { School Canteen Guidelines [6] }\end{array}$ & $\begin{array}{l}\text { Mandatory for public } \\
\text { schools, voluntary for } \\
\text { independent schools }\end{array}$ & $N S, I, G, R$ & Yes & $\begin{array}{l}\text { Annual. Full review to } \\
\text { be conducted within a } \\
3 \text { year period (date not } \\
\text { specified) }\end{array}$ \\
\hline New South Wales; 2018 & $\begin{array}{l}\text { Nutrition in Schools } \\
\text { Policy }[24,71-75]\end{array}$ & $\begin{array}{l}\text { Mandatory for public } \\
\text { schools, voluntary for } \\
\text { independent schools }\end{array}$ & $N S, I, G, R, M / E$ & Yes & $\begin{array}{l}\text { Every } 3 \text { years (date not } \\
\text { specified) }\end{array}$ \\
\hline Northern Territory; 2017 & $\begin{array}{l}\text { School Nutrition and Healthy } \\
\text { Eating Policy [25, 76-84] / } \\
\text { National Healthy School } \\
\text { Canteen Guidelines [6] }\end{array}$ & $\begin{array}{l}\text { Mandatory for public } \\
\text { schools }\end{array}$ & $N S, G, R$ & Yes & No \\
\hline Queensland; 2016 & $\begin{array}{l}\text { Smart Choices. Healthy food and } \\
\text { drink supply strategy for Queensland } \\
\text { schools }[85,86]\end{array}$ & $\begin{array}{l}\text { Mandatory for public } \\
\text { schools }\end{array}$ & $N S, I, G, R, M / E$ & No & No \\
\hline $\begin{array}{l}\text { South Australia; } 2015 \\
\text { (review planned) }\end{array}$ & $\begin{array}{l}\text { Right Bite, Healthy Food and Drink } \\
\text { Supply Strategy for South Australian } \\
\text { Schools and Preschools [87-90] }\end{array}$ & $\begin{array}{l}\text { Mandatory for public } \\
\text { schools, voluntary for } \\
\text { independent schools }\end{array}$ & $N S, I, R, M / E$ & Yes & No \\
\hline Tasmania; 2014 & $\begin{array}{l}\text { Tasmania School Canteen Handbook. } \\
\text { A Whole School Approach to Healthy } \\
\text { Eating [91]/ National Healthy School } \\
\text { Canteen Guidelines [6] }\end{array}$ & Voluntary & $N S, I, G, R, M / E, A P$ & No & No \\
\hline Victoria; 2006 & Healthy Canteen Kit [92-95] & $\begin{array}{l}\text { Mandatory for public } \\
\text { schools }\end{array}$ & $N S, I, G, R, A P$ & Yes & No \\
\hline Western Australia; 2017 & $\begin{array}{l}\text { Healthy Food and Drink } \\
\text { Policy }[26,96-99]\end{array}$ & $\begin{array}{l}\text { Mandatory for public } \\
\text { schools }\end{array}$ & $N S, I, G, R, A P$ & Yes & No \\
\hline \multicolumn{6}{|l|}{ Workplaces } \\
\hline $\begin{array}{l}\text { Australian Capital } \\
\text { Territory; } 2015\end{array}$ & Healthier work [100-107] & Voluntary & $V T, I, R, M / E, A P$ & Recommended & $\begin{array}{l}\text { Recommended: } \\
6 \text { months, then } \\
\text { annually (qualitative) }\end{array}$ \\
\hline New South Wales; 2017 & Get Healthy At Work [108-111] & Voluntary & $N S, G, R$ & No & No \\
\hline Northern Territory; 2017 & $\begin{array}{l}\text { The Healthy Workplace } \\
\text { Toolkit [112] }\end{array}$ & Voluntary & $N S, V T, G, R, M / E$ & No & No \\
\hline Queensland; 2013 & $\begin{array}{l}\text { Healthier. Happier. } \\
\text { Workplaces [113-117] }\end{array}$ & Voluntary & $V T, I, R, M / E, A P$ & No & No \\
\hline
\end{tabular}


Table 1 Comparison of the design and components of state and territory nutrition policies in Australian institutions (Continued)

\begin{tabular}{|c|c|c|c|c|c|}
\hline $\begin{array}{l}\text { State/territory and year } \\
\text { introduced or most } \\
\text { recently updated }\end{array}$ & Name of policy/guideline & Approach & Components & $\begin{array}{l}\text { Outline } \\
\text { of roles/ } \\
\text { responsibilities }\end{array}$ & $\begin{array}{l}\text { Outline of monitoring } \\
\text { and evaluation plan }\end{array}$ \\
\hline South Australia; 2014 & $\begin{array}{l}\text { A Workplace Health and Wellbeing } \\
\text { Toolkit: Step by Step Guide to } \\
\text { Developing a Successful } \\
\text { Workplace Program [118] }\end{array}$ & Voluntary & $V T, I, R, M / E$ & No & No \\
\hline Tasmania; 2013 & $\begin{array}{l}\text { Healthy Workplace Nutrition } \\
\text { Guidelines [119-121] }\end{array}$ & Voluntary & $N S, V T, I, G, R$ & No & No \\
\hline Victoria; 2016 & $\begin{array}{l}\text { Healthy choices: healthy eating } \\
\text { policy and catering guide for } \\
\text { workplaces }[54,122]\end{array}$ & Voluntary & $N S, V T, I, G, R, A P$ & Yes & $\begin{array}{l}\text { Recommended: } 6 \\
\text { months, then annually } \\
\text { (qualitative) }\end{array}$ \\
\hline Western Australia; 2017 & Healthier Workplace WA [123-132] & Voluntary & $N S, V T, I, G, R, M / E, A P$ & No & Nil \\
\hline \multicolumn{6}{|l|}{ Other settings } \\
\hline Victoria; 2016 & $\begin{array}{l}\text { Healthy choices: policy guidelines } \\
\text { for parks }[54,133]\end{array}$ & Voluntary & $N S, I, G, R$ & No & $\begin{array}{l}\text { Recommended: } 12 \\
\text { month review only } \\
\text { (qualitative) }\end{array}$ \\
\hline $\begin{array}{l}\text { Australian Capital } \\
\text { Territory; } 2016\end{array}$ & $\begin{array}{l}\text { ACT Public Sector: Healthy Food } \\
\text { and Drink Choices Policy and } \\
\text { Vending Machine Management } \\
\text { Policy }[31,32,134,135]\end{array}$ & Mandatory & $N S, G, R$ & Yes & Nil \\
\hline Queensland: 2014 & $\begin{array}{l}\text { Choose Well, Live Well - Remote } \\
\text { Area Camp Food [136] }\end{array}$ & Voluntary & $N S, I, R, E$ & No & Nil \\
\hline Victoria; 2016 & $\begin{array}{l}\text { Healthy choices: policy guidelines } \\
\text { for sport and recreation } \\
\text { centres }[54,137]\end{array}$ & Voluntary & $N S, V T, I, G, R$ & Yes & $\begin{array}{l}\text { Recommended: } 6 \\
\text { months, then annually } \\
\text { (qualitative) }\end{array}$ \\
\hline
\end{tabular}

ACT Australian Capital Territory, NSW New South Wales, NT Northern Territory, SA South Australia, WA Western Australia, NS Nutrition standards, VT Voluntary policy template, I Implementation guide/tools, $G$ Guide for catering/fundraising/advertising, $R$ Extra resources to support implementation, $M / E$ Monitoring/ Evaluation guide/tools, AP Accreditation program

The percentages of different foods/drinks permitted in food outlets/canteens and vending, and for catering, fundraising and advertising, varied between jurisdictions and between types of institutions. In food outlets/canteens and vending, policies specified frequencies of specific foods permitted, including minimum proportions of: 'green' or 'green and amber' traffic-light classified foods/drinks, or 'everyday' foods; and/or maximum proportions of 'red' and 'amber' traffic-light classified foods or 'occasional' foods. In New South Wales, a minimum of $75 \%$ 'everyday' and maximum of $25 \%$ 'occasional' foods/drinks were permitted across all institution types, and there was a ban on sugary drinks in schools and health facilities. In the other jurisdictions, permitted proportions ranged from no 'red' food/drinks in most schools, to less than $20 \%$ 'red' in other institutions. Proportions of 'green' foods ranged from 50 to $60 \%$ in these jurisdictions, however many simply specified 'majority' to be 'green' and others specified a minimum of $80 \%$ combined 'green and amber' foods/drinks. For catering, fundraising and advertising, policies specified targets such as majority 'green', only 'green' and no 'red', or only 'everyday'. Full details are displayed in Table 2.

\section{Comparison of nutrient criteria}

The majority of policies specified that the nutrient criteria were adapted from other jurisdictions. Most were directly based on the Fresh Tastes @ School NSW
Healthy School Canteen Strategy, or were adaptations [139]. Other policies adopted the National Healthy School Canteen Guidelines [6] or applied criteria based on the national guidelines.

The number of food categories targeted varied by jurisdiction, from seven in the South Australian school policy to 45 in the New South Wales health facility policy. Despite the large number of food categories targeted in many jurisdictions, few were consistently targeted across all. Overall, only five food categories were targeted as part of nutrient criteria by every state and territory in all three institution types: meat products (e.g. crumbed and coated foods, sausages, frankfurts and saveloys), hot food items (e.g. savoury pastry, pizza, oven-baked potato products, instant noodles), cakes/sweet tarts/pastries, ice-creams and dairy desserts and sweet snacks (e.g. muesli bars, sweet biscuits).

In health facilities, eight food categories were targeted by every jurisdiction with a policy, while 15 appeared in the policy of more than one state or territory. In schools, six were targeted by every jurisdiction, while 20 food categories appeared in more than one state or territory policy. For work places, seven were targeted by every jurisdiction, while 15 appeared in more than one state or territory policy. Of these commonly targeted food categories, the majority of energy and fibre criteria were consistent between jurisdictions, however only around half of the 
Table 2 Comparison of the design of state and territory level procurement policies in Australian institutions

\begin{tabular}{llll}
\hline $\begin{array}{l}\text { Jurisdictions and year } \\
\text { introduced or updated }\end{array}$ & $\begin{array}{l}\text { Name of Procurement Policy/ } \\
\text { Nutrient Standards }\end{array}$ & $\begin{array}{l}\text { System } \\
\text { of different foods permitted } \\
\text { for food outlets/ canteens } \\
\text { and vending }\end{array}$ & $\begin{array}{l}\text { Percentages or frequency of } \\
\text { different foods permitted for catering, } \\
\text { fundraising and } \\
\text { advertising }\end{array}$
\end{tabular}

\section{Health Facilities}

Australian Capital

Territory; 2013

Healthy Food and Drink Choices Policy

New South Wales; 2017

Northern Territory; 2017

Queensland; 2007

South Australia; 201

Tasmania

Victoria; 2016

Western Australia; 2017

\section{Schools}

Australian Capital Territory; 2015

New South Wales; 2017

Northern Territory; 2017

Queensland; 2016 revised

South Australia; 2004

Tasmania; 2014
Healthy Food and Drink Choices for Staff and Visitors in SA Health Facilities Policy

Nil

Healthy Choices: Food and Drink Classification Guide

The Healthy Options WA: Making Healthy Food Choices Easier Implementation Guide

Healthy Food and Drink in NSW Health Facilities for Staff and Visitors Framework

Food and Drink for Staff, Volunteers and Visitors in Territory Health Facilities: Healthy Choices Made Easy Foods and Drink Supply Strategy for OLD Health Facilities

ational Healthy School Canteen Guidelines

The NSW Healthy School Canteen Strategy. Food and Drink Criteria

National Healthy School Canteen Guidelines

Smart Choices. Healthy Food and Drink Supply Strategy for Queensland Schools.

Right Bite. Healthy Food and Drink Supply Strategy for South Australian Schools and Preschools

National Healthy School Canteen Guidelines (2014)

TL Food Outlets - Green: Majority, Green and Amber: $\geq 80 \%$, Red: $<20 \%$.

Vending - Red: $<20 \%$.

HSR

Food Outlets and Vending Everyday: $\geq 75 \%$, Occasional: $<25 \%$. Ban on sugary drinks.

TL Food Outlets and Vending Green and Amber: $\geq 80 \%$ (Aim for $\geq 50 \%$ of green). Red: $<20 \%$.

TL Food Outlets - Green and Amber: $\geq 80 \%$. Red: < 20\%. Over time increase to $\geq 50 \%$ green choices.

Vending - Red: Nil.

Food Outlets and Vending Red: $<20 \%$

$\mathrm{Nil}$

Food Outlets and Vending Green: $\geq 50 \%$, Red: <20\%.

Food Outlets and Vending Green: $\geq 50 \%$, Red: <20\%.

Food Outlets - Green: Majority Red: Nil. Ban on sugary drinks. Vending: Nil permitted.

Food Outlets and Vending Everyday: $\geq 75 \%$, Occasional: $\leq 25 \%$. Ban on sugary drinks.

Food Outlets and Vending Green: Majority, Red: Nil.

Food Outlets and Vending Red: $\leq$ two occasions per term.

Food Outlets and Vending Green: Majority, Red: Nil.

Food Outlets and Vending Green: Majority, Red: Nil.
Catering - Green: Majority. Red: Nil.

Fundraising - Green: Majority. Red: Nil.

Advertising - Green: Only.

Catering - Everyday: $\geq 75 \%$, Occasional: $<25 \%$

Advertising - Everyday: only.

Catering - Red: Nil.

Fundraising - Red: Nil.

Advertising - Green: Only.

Catering - Red: Nil.

Fundraising - Red: Nil.

Advertising - Green: Only.

Catering - Red: Nil.

Fundraising - Red: Nil.

Advertising - Red: Nil.

Nil

Catering - Green: Majority, Amber: Small quantities only. Red: Nil.

Fundraising - Red: Nil.

Advertising - Green: Majority, Red: Nil.

Catering - Green: $\geq 50 \%$

Red: $<20 \%$.

Fundraising - Green: $\geq 50 \%$,

Red: $<20 \%$.

Advertising - Green: Only.

Fundraising: $\leq$ two occasions per term.

Advertising - Everyday: Only.

Catering - Green: Majority, Red: Nil. Fundraising - Green: Majority, Red: Nil.

Advertising - Green: Only.

Fundraising - Red: $\leq$ two occasions per term.

Advertising - Green: Only.

Catering - Red: $\leq$ two occasions per term.

Advertising - Green: Only.

Catering - Green: Majority, Red: Nil. Fundraising - Green: Majority, Red: Nil.

Advertising - Green: Only. 
Table 2 Comparison of the design of state and territory level procurement policies in Australian institutions (Continued)

\begin{tabular}{|c|c|c|c|c|}
\hline $\begin{array}{l}\text { Jurisdictions and year } \\
\text { introduced or updated }\end{array}$ & $\begin{array}{l}\text { Name of Procurement Policy/ } \\
\text { Nutrient Standards }\end{array}$ & System & $\begin{array}{l}\text { Percentages or frequency } \\
\text { of different foods permitted } \\
\text { for food outlets/ canteens } \\
\text { and vending }\end{array}$ & $\begin{array}{l}\text { Percentages or frequency of } \\
\text { different foods permitted for catering, } \\
\text { fundraising and } \\
\text { advertising }\end{array}$ \\
\hline $\begin{array}{l}\text { Victoria; } 2006 \\
\text { (currently being } \\
\text { revised) }\end{array}$ & Healthy Canteen Kit. Food Planner & $\mathrm{TL}$ & $\begin{array}{l}\text { Food Outlets - Green: Majority, } \\
\text { Red: Nil. } \\
\text { Vending - Red: Nil. Ban on } \\
\text { sugary drinks and confectionary. }\end{array}$ & $\begin{array}{l}\text { Catering - Green: Majority, Red: } \\
\leq \text { two occasions per term. } \\
\text { Advertising - Green: Majority, } \\
\text { Amber: Minority. }\end{array}$ \\
\hline $\begin{array}{l}\text { Western Australia; } \\
2017\end{array}$ & $\begin{array}{l}\text { Federation of Canteens in Schools } \\
\text { (FOCiS) Food Industry Handbook }\end{array}$ & $\mathrm{TL}$ & $\begin{array}{l}\text { Food Outlets - Green: } \geq 60 \% \text {. } \\
\text { Amber: < 40\%. Red: Nil. }\end{array}$ & $\begin{array}{l}\text { Catering - Red: Nil. } \\
\text { Fundraising - Red: Nil. }\end{array}$ \\
\hline \multicolumn{5}{|l|}{ Workplaces } \\
\hline $\begin{array}{l}\text { Australian Capital } \\
\text { Territory; } 2015\end{array}$ & Nil & Nil & $\mathrm{Nil}$ & Nil \\
\hline $\begin{array}{l}\text { New South Wales; } \\
2017\end{array}$ & $\begin{array}{l}\text { Get Healthy at Work. Healthier } \\
\text { Food and Drinks Guide }\end{array}$ & $\mathrm{HSR}$ & $\begin{array}{l}\text { Food Outlets and Vending - } \\
\text { Everyday: } \geq 75 \% \text {, Occasional: } \\
\leq 25 \% \text {. }\end{array}$ & $\begin{array}{l}\text { Catering - Everyday: } \geq 75 \% \text {, } \\
\text { Occasional: } \leq 25 \% \text {. }\end{array}$ \\
\hline $\begin{array}{l}\text { Northern Territory; } \\
2017\end{array}$ & $\begin{array}{l}\text { The Healthy Workplace Toolkit: } \\
\text { Healthy Workplace Nutrition } \\
\text { Guidelines }\end{array}$ & $\mathrm{TL}$ & $\begin{array}{l}\text { Food Outlets and Vending } \\
\text { Recommendation - Green: } \\
\geq 50 \% \text {, Red: < 20\%). }\end{array}$ & $\begin{array}{l}\text { Catering - Red: Limited. } \\
\text { Fundraising - Red: Limited. }\end{array}$ \\
\hline Queensland; 2013 & Nil & Nil & $\mathrm{Nil}$ & Nil \\
\hline South Australia; 2014 & Nil & Nil & $\mathrm{Nil}$ & Nil \\
\hline Tasmania; 2013 & $\begin{array}{l}\text { Healthy Workplace Nutrition } \\
\text { Guidelines }\end{array}$ & $\mathrm{TL}$ & $\mathrm{Nil}$ & Nil \\
\hline Victoria; 2016 & $\begin{array}{l}\text { Healthy Choices: Food and Drink } \\
\text { Classification Guide }\end{array}$ & $\mathrm{TL}$ & Vending - Red: $\leq 20 \%$ & $\begin{array}{l}\text { Catering - Green: Majority, } \\
\text { Red: Nil. } \\
\text { Fundraising - Red: } \\
\text { Nil for children. } \\
\text { Advertising - } \\
\text { Green: Majority, Red: Nil. }\end{array}$ \\
\hline $\begin{array}{l}\text { Western Australia; } \\
2017\end{array}$ & $\begin{array}{l}\text { Healthy Choices: Healthy Futures } \\
\text { Healthier Food and Drinks Guide }\end{array}$ & Nil & $\begin{array}{l}\text { Food Outlets and Vending } \\
\text { recommendations - Green: } \\
\geq 50 \% \text {, Red: }<20 \% \text {. }\end{array}$ & $\begin{array}{l}\text { Catering recommendations - } \\
\text { Green: } \geq 50 \% \text {, Red: }<20 \% \text {. }\end{array}$ \\
\hline \multicolumn{5}{|l|}{ Other settings } \\
\hline Victoria; 2016 & $\begin{array}{l}\text { Healthy Choices: Food and Drink } \\
\text { Classification Guide }\end{array}$ & $\mathrm{TL}$ & $\begin{array}{l}\text { Food Outlets and Vending - } \\
\text { Green: } \geq 50 \% \text {, Red: }<20 \% \text {. }\end{array}$ & $\begin{array}{l}\text { Catering - Green: Majority, } \\
\text { Red: Nil. } \\
\text { Fundraising - Red: } \\
\text { Nil for children and youth. } \\
\text { Advertising - Green: Majority, } \\
\text { Red: Nil. }\end{array}$ \\
\hline $\begin{array}{l}\text { Australian Capital } \\
\text { Territory; } 2016\end{array}$ & $\begin{array}{l}\text { Healthy Food and Drink Choices } \\
\text { Policy - Public Sector }\end{array}$ & $\mathrm{TL}$ & $\begin{array}{l}\text { Food Outlets - Green: } \\
\text { Majority, Green and } \\
\text { Amber: } \geq 80 \% \text {. }\end{array}$ & $\begin{array}{l}\text { Catering - Green: Majority, } \\
\text { Red: Nil. } \\
\text { Fundraising - Green: } \\
\text { Majority, Red: Nil. } \\
\text { Advertising - Green: Only. }\end{array}$ \\
\hline Queensland: 2014 & $\begin{array}{l}\text { Choose Well, Live Well - Remote } \\
\text { Area Camp Food }\end{array}$ & $\mathrm{TL}$ & $\mathrm{Nil}$ & $\begin{array}{l}\text { Catering - Green: } \geq 50 \% \text {. Amber: } \\
<30 \% \text {. Red }<20 \% \text {. }\end{array}$ \\
\hline Victoria; 2016 & $\begin{array}{l}\text { Healthy Choices: Food and Drink } \\
\text { Classification Guide }\end{array}$ & $\mathrm{TL}$ & $\begin{array}{l}\text { Food Outlets and Vending - } \\
\text { Green: } \geq 50 \% \text {, Red: }<20 \% \text {. }\end{array}$ & $\begin{array}{l}\text { Catering - Green: Majority, } \\
\text { Red: Nil. } \\
\text { Fundraising - Red: } \\
\text { Nil for children and youth. } \\
\text { Advertising - Green: Majority, } \\
\text { Red: Nil. }\end{array}$ \\
\hline
\end{tabular}

NSW New South Wales, QLD Queensland, SA South Australia, WA Western Australia, TL traffic light, HSR Health Star Rating

saturated fat cut-offs and less than half of the sodium criteria were consistent. For example, sodium criteria for meat products varied across all three settings, ranging from $\leq 450 \mathrm{mg}$ to $700 \mathrm{mg} / 100 \mathrm{~g}$. There were also discrepancies in saturated fat criteria across a range of food categories, with some jurisdictions applying a grams per serve cut-off and others specifying the same cut-off $(\mathrm{g})$ per $100 \mathrm{~g}$. A detailed summary of foods with nutrient criteria in all jurisdictions is displayed in Table 3. 
Table 3 Summary of amber nutrient criteria for commonly targeted food categories in Australian institutions

\begin{tabular}{|c|c|c|c|c|c|}
\hline Food & Energy & Saturated Fat & Sodium & Sugar & Fibre \\
\hline \multicolumn{6}{|c|}{ Health Facilities (ACT, NT, QLD, SA, VIC, WA) } \\
\hline Cakes/sweet tarts/pastries & $\leq 900 \mathrm{~kJ} / \mathrm{serve}$ & $\begin{array}{l}\leq 3 \mathrm{~g} / \text { serve } \\
(\leq 3 / 100 \mathrm{~g} \mathrm{SA}, \mathrm{NT})\end{array}$ & $\leq 300 \mathrm{mg} / \mathrm{serve}$ (WA only) & - & $\geq 1.5 \mathrm{~g} / \mathrm{serve}$ \\
\hline $\begin{array}{l}\text { Savoury snacks (e.g. savoury } \\
\text { biscuits, crisps) }\end{array}$ & $\begin{array}{l}\leq 600 \mathrm{~kJ} / \text { serve (and } \\
\text { some states } \leq 1800 \mathrm{~kJ} / \\
100 \mathrm{~g} \text { ) }\end{array}$ & $\begin{array}{l}\leq 2-3 \mathrm{~g} / \text { serve } \\
(\leq 3 \mathrm{~g} / 100 \mathrm{~g} \mathrm{SA}, \mathrm{NT})\end{array}$ & $\leq 200 \mathrm{mg} / \mathrm{serve}$ & - & - \\
\hline $\begin{array}{l}\text { Processed meat (cold luncheon } \\
\text { and cured meats) }\end{array}$ & $\leq 900-1000 \mathrm{~kJ} / 100 \mathrm{~g}$ & $\leq 3 \mathrm{~g} / 100 \mathrm{~g}$ & $\begin{array}{l}\leq 750 \mathrm{mg} / 100 \mathrm{~g}(\mathrm{ACT} \\
\text { VIC, WA only) }\end{array}$ & - & - \\
\hline $\begin{array}{l}\text { Meat products (e.g. crumbed and } \\
\text { coated meat, frankfurts) }\end{array}$ & $\leq 1000 \mathrm{~kJ} / 100 \mathrm{~g}$ & $\leq 5 \mathrm{~g} / 100 \mathrm{~g}$ & $\leq 450-700 \mathrm{mg} / 100 \mathrm{~g}$ & - & - \\
\hline $\begin{array}{l}\text { Hot food items (e.g. savoury pastries, } \\
\text { pizza, instant noodles) }\end{array}$ & $\leq 1000 \mathrm{~kJ} / 100 \mathrm{~g}$ & $\leq 5 \mathrm{~g} / 100 \mathrm{~g}$ & $\leq 400 \mathrm{mg} / 100 \mathrm{~g}$ & - & - \\
\hline Pre-prepared meals & $\leq 2500 \mathrm{~kJ} /$ serve & $\begin{array}{l}\leq 2 \mathrm{~g} / 100 \mathrm{~g} \\
(\leq 2 \mathrm{~g} / \text { serve VIC) }\end{array}$ & $\begin{array}{l}\leq 900 \mathrm{mg} / \text { serve }+ \\
\leq 300 \mathrm{mg} / 100 \mathrm{~g}\end{array}$ & - & $\geq 3 \mathrm{~g} /$ serve \\
\hline Ice-creams and dairy desserts & $\leq 600 \mathrm{~kJ} / \mathrm{serve}$ & $\begin{array}{l}\leq 3 \mathrm{~g} / \text { serve } \\
(\leq 3 \mathrm{~g} / 100 \mathrm{~g} \mathrm{NT})\end{array}$ & - & - & - \\
\hline $\begin{array}{l}\text { Sweet snacks (e.g. muesli bars, sweet } \\
\text { biscuits). }\end{array}$ & $\leq 600 \mathrm{~kJ} /$ serve & $\begin{array}{l}\leq 3 \mathrm{~g} / 100 \mathrm{~g} \\
(\leq 3 \mathrm{~g} / \text { serve VIC) }\end{array}$ & - & - & $\geq 1 \mathrm{~g} /$ serve \\
\hline \multicolumn{6}{|c|}{ Schools (ACT, NT, QLD, SA, TAS, VIC, WA) ${ }^{a}$} \\
\hline $\begin{array}{l}\text { Meat products (e.g. crumbed and } \\
\text { coated foods, sausages, frankfurts) }\end{array}$ & $\leq 1000 \mathrm{~kJ} / 100 \mathrm{~g}$ & $\leq 5 \mathrm{~g} / 100 \mathrm{~g}$ & $\leq 450-700 \mathrm{mg} / 100 \mathrm{~g}$ & - & - \\
\hline $\begin{array}{l}\text { Hot food items (e.g. savoury pastry, } \\
\text { pizza, oven-baked potato products, } \\
\text { instant noodles) }\end{array}$ & $\leq 1000 \mathrm{~kJ} / 100 \mathrm{~g}$ & $\leq 5 \mathrm{~g} / 100 \mathrm{~g}$ & $\leq 400 \mathrm{mg} / 100 \mathrm{~g}$ & - & - \\
\hline Cakes/sweet tarts/pastries & $\leq 900 \mathrm{~kJ} /$ serve & $\begin{array}{l}\leq 3 \mathrm{~g} / \text { serve } \\
(\leq 3 \mathrm{~g} / 100 \mathrm{~g} W A)\end{array}$ & - & - & $\geq 1.5 \mathrm{~g} /$ serve \\
\hline $\begin{array}{l}\text { Savoury snacks (e.g. savoury biscuits, } \\
\text { crisps) }\end{array}$ & $\begin{array}{l}\leq 600 \mathrm{~kJ} / \text { serve }(\text { and } \\
\text { some states } \\
\leq 1800 \mathrm{~kJ} / 100 \mathrm{~g} \text { ) }\end{array}$ & $\leq 2 \mathrm{~g} /$ serve & $\leq 200 \mathrm{mg} / \mathrm{serve}$ & - & - \\
\hline Ice-creams and dairy desserts & $\leq 600 \mathrm{~kJ} / \mathrm{serve}$ & $\leq 3 \mathrm{~g} /$ serve & - & - & - \\
\hline $\begin{array}{l}\text { Sweet snacks (e.g. muesli bars, } \\
\text { sweet biscuits) }\end{array}$ & $\leq 600 \mathrm{~kJ} /$ serve & $\leq 3 \mathrm{~g} /$ serve & - & - & $\geq 1 \mathrm{~g} /$ serve \\
\hline \multicolumn{6}{|l|}{ Workplaces (NT, TAS, VIC, WA) } \\
\hline Cakes/sweet tarts/pastries & $\leq 600-900 \mathrm{~kJ} /$ serve & $\begin{array}{l}\leq 3 \mathrm{~g} / 100 \mathrm{~g}(\leq 3 \mathrm{~g} / \\
\text { serve VIC, WA })\end{array}$ & $\begin{array}{l}\leq 200 \mathrm{mg} / \mathrm{serve} \\
\text { (TAS only) }\end{array}$ & $\begin{array}{l}5-15 \mathrm{~g} / 100 \mathrm{~g} \\
\text { (WA only) }\end{array}$ & $\geq 1.5 \mathrm{~g} /$ serve \\
\hline $\begin{array}{l}\text { Processed meat (cold luncheon and } \\
\text { cured meats) }\end{array}$ & $\begin{array}{l}\leq 900-1000 \mathrm{~kJ} / 100 \mathrm{~g} \\
(\leq 600 \mathrm{~kJ} / \mathrm{serve} W A)\end{array}$ & $\leq 3 \mathrm{~g} / 100 \mathrm{~g}$ & $\leq 700-750 \mathrm{mg} / 100 \mathrm{~g}$ & - & - \\
\hline $\begin{array}{l}\text { Meat products (e.g. crumbed and } \\
\text { coated meat, frankfurts) }\end{array}$ & $\leq 1000 \mathrm{~kJ} / 100 \mathrm{~g}$ & $\leq 5 \mathrm{~g} / 100 \mathrm{~g}$ & $\leq 450-700 \mathrm{mg} / 100 \mathrm{~g}$ & - & - \\
\hline $\begin{array}{l}\text { Hot food items (e.g. savoury pastries, } \\
\text { pizza, instant noodles) }\end{array}$ & $\leq 1000 \mathrm{~kJ} / 100 \mathrm{~g}$ & $\leq 5 \mathrm{~g} / 100 \mathrm{~g}$ & $\leq 400 \mathrm{mg} / 100 \mathrm{~g}$ & - & - \\
\hline Pre-prepared meals & $\begin{array}{l}\leq 2500 \mathrm{~kJ} / \text { serve } \\
\text { (nil WA) }\end{array}$ & $\begin{array}{l}\leq 2 \mathrm{~g} / 100 \mathrm{~g} \text { VIC, } \leq 2 \\
\mathrm{~g} / \text { serve NT, } \leq 3 \mathrm{~g} / \\
\text { serve WA, } \leq 4 \mathrm{~g} / 100 \mathrm{~g} \\
\text { TAS }\end{array}$ & $\begin{array}{l}\leq 900 \mathrm{mg} / \mathrm{serve} \text { and } \\
\leq 300 \mathrm{mg} / 100 \mathrm{~g} \\
(400 \mathrm{mg} / 100 \mathrm{~g} \mathrm{WA})\end{array}$ & - & $\geq 3 \mathrm{~g} /$ serve \\
\hline Ice-creams and dairy desserts & $\leq 600 \mathrm{~kJ} / \mathrm{serve}$ & $\begin{array}{l}\leq 3 \mathrm{~g} / 100 \mathrm{~g}(\leq 3 \mathrm{~g} / \\
\text { serve VIC, WA })\end{array}$ & - & $\begin{array}{l}5-15 \mathrm{~g} / 100 \mathrm{~g} \\
\text { (WA only) }\end{array}$ & - \\
\hline $\begin{array}{l}\text { Sweet snacks (e.g. muesli bars, } \\
\text { sweet biscuits). }\end{array}$ & $\leq 600 \mathrm{~kJ} / \mathrm{serve}$ & $\begin{array}{l}\leq 3 \mathrm{~g} / 100 \mathrm{~g}(\leq 3 \mathrm{~g} / \\
\text { serve VIC, WA })\end{array}$ & - & $\begin{array}{l}5-15 \mathrm{~g} / 100 \mathrm{~g} \\
\text { (WA only) }\end{array}$ & $\begin{array}{l}\geq 1 \mathrm{~g} / \text { serve } \\
(\geq 3 \mathrm{~g} / \text { serve WA })\end{array}$ \\
\hline
\end{tabular}

${ }^{a}$ New South Wales policies were excluded since criteria are based on portion size and Health Star Rating, rather than traffic light labelling. ACT Australian Capital Territory, NT Northern Territory, QLD Queensland, SA South Australia, TAS Tasmania, VIC Victoria, WA Western Australia 


\section{Discussion}

The large number of state and territory level institutional nutrition policies and guidelines in Australia highlights the perceived importance of these across the nation. This study found that all eight Australian jurisdictions have school and workplace nutrition policies/ guidelines, seven have a health facility policy, and a further four policies were identified for other settings. Yet, there were notable differences in policy design and components, between institution types and jurisdictions, which may affect policy implementation, and potential impact. As an example, three-quarters of policies included implementation guides or tools, however less than half outlined procedures for monitoring and evaluation, which is a key component of any well-designed policy [140, 141]. In addition, important differences in nutrient criteria were revealed that may also be limiting policy impact. Inconsistencies between jurisdictions and institution types reveal a potential challenge for manufacturers attempting to reformulate in line with multiple different policies, and illustrates the scope for aligning and strengthening nutrition policies.

Most health facility and school nutrition policies in Australia are mandatory for government institutions and include nutrition standards. There is existing evidence that these policies can have a positive impact on dietary intake [11], though to be successful, policies must be well designed, include a number of key components, and have systems in place to support implementation, monitoring and evaluation [13]. Poor compliance in implementing policies in schools and health facilities $[20,28]$ has previously been documented as a major concern and has likely hindered the potential impact in Australia. A number of reasons for this have been proposed, some of which include: complexity of the product categories, concerns about loss of profit [142], lack of organisational leadership [143], lack of assistance with implementation [144], and difficulty sourcing appropriate foods [27]. Other reasons from global literature include: ambiguity in policy wording, inadequate human and financial resources, a lack of accountability mechanisms for effective monitoring and policy enforcement, and conflicts of interest between institutions and private partnerships [145]. This global evidence suggests gaps in the policy design during planning processes may also present a barrier to implementation. Our study illustrated that the majority of Australian policies contained components which are key to success, including resources to support implementation and defined roles and responsibilities $[140,141]$. Yet, gaps in accountability mechanisms, such as a lack of timelines and tools for monitoring and evaluation in many jurisdictions, highlight a crucial area for improvement and suggest another reason for the poor compliance.
In contrast to the health and school policies, workplace nutrition policies are all voluntary. More importantly, only half have voluntary nutrient standards included, a gap that makes the policies difficult to operationalise. Almost all policies encouraged workplaces to develop their own guidelines and strategies based on health promoting principles by providing a policy template to modify, as opposed to adopting a state/territorywide policy (e.g. [100, 123]). With the exception of Victoria, all workplace policies targeted a variety of noncommunicable disease risk factors, such as physical activity and smoke-free environments, in addition to healthy eating. Further, the healthy eating components tended to focus on generic healthy eating strategies, such as replacing biscuits with fresh fruit [100], or displaying posters with health promotion messages [123]. The flexible policy design may incentivise workplaces to create a policy with healthy eating components, however the lack of structure and set guidance for food procurement, preparation and provision likely lessens any potential impact. Positively, many policies did include tools or resources for monitoring and evaluation, however roles and responsibilities were less likely to be defined, possibly due to the large variation in workplace sizes and structures. Similar to other institution types, the absence of this accountability mechanism may also be a barrier to implementation and monitoring of these policies.

The identification of policies in other settings illustrates large potential for future work nationwide. The Australian Food-EPI reports [29] highlight establishing a whole-of-government policy with nutrition standards to be a key next step in this area. A whole-of-government approach would apply to all government departments, and settings under government control [146]. So far, the Australian Capital Territory is the only jurisdiction with a policy for the public sector, though still retains individual policies for schools, workplaces and health facilities. However, work towards a public sector policy is underway in Victoria, and being planned in Western Australia [29]. In addition, Victoria has two unique policies targeting retailers in parks and sport/recreation centres that are under government control. These policies are relevant to all Australian jurisdictions and could be adopted or adapted for use within each jurisdiction, creating healthy food environments across a wider range of settings [7]. Further, developing a whole-of-government approach, applying across all public institutions and public settings, could eliminate the inconsistencies between policies for different institution types.

The design of institutional nutrition policies varies globally [17], depending on factors such as governance, food systems, and methods of providing and selling foods (e.g. food outlets, vending, catering and fundraising). A framework for monitoring and evaluating 
nutrition standards in publicly-funded institutions, based on a review of known strategies, highlights the variability in policy design [13]. In countries such as Australia and Canada, institutional nutrition policies are the responsibility of states/provinces and territories [14, 147], while in other countries these may be implemented at the national level (e.g. England [148]), or city/municipal/local government level (e.g. New York City [149]). While Australian policies are 'nutrient-based', other policies such as in schools in the UK are food group-based [17]. A concern with the Australian approach is that nutrientbased standards may simply lead to replacement of foods with similar foods which are lower in adverse nutrients (e.g. use sweetener instead of sugar) but still of poor nutritional value, as seen in California [150]. A combination of food and nutrient-based standards is recommended to prevent this issue occurring [13], and including food-based standards in the Australian policies could increase their impact on dietary intake. In terms of application of the policies, most Australian jurisdictions use an 'in/out' approach based on the nutrient criteria, in combination with categories to 'choose most/ least'. However, the New South Wales policy uses the HSR [5], a composite score approach, in combination with portion size strategies. Other countries also apply one or more of these strategies in their institutional nutrition policies, for example in schools, Bahrain uses an 'in/out' approach, Malaysia uses a 'choose most/least' approach, and Mexico uses a combination of the two [151]. Another area of difference is that Australian school policies apply to foods provided and sold, but do not include standards for the composition of meals, as seen in some policies, for example in schools in Sweden [17, 151], as Australian schools do not provide lunch meals for students. Using L'Abbé et al.'s monitoring and evaluation framework [13] to compare policies globally could provide useful insights into strengthening current institutional policies, but is outside the scope of the current paper.

The complex nature of the nutrient criteria in institutional nutrition policies, where they exist, has been previously identified as a barrier to implementation [142], and variations in criteria between jurisdictions may also be a hindrance [152]. The criteria are complex in that multiple foods and food categories are clustered together, and the wide variety of products within each group may result in challenges for institutions in procuring, preparing or providing foods meeting these criteria. For example, all meat products and alternatives are grouped together in most jurisdictions, meaning the same nutrient criteria are applied to a chicken nuggets and sausages. Yet, there are known differences in the nutrient composition of these products (e.g. sodium content [153, 154]), and individually set targets may be more appropriate. The variations between criteria for similar categories between states may also be a barrier [152], particularly as many manufacturers tend to supply food nationally, rather than within a particular jurisdiction, and therefore must manufacture products to meet the most stringent jurisdiction's nutrient criteria. The challenges associated with the design of the institutional nutrition policies can be contrasted with the design of the national nutrient reformulation targets proposed by the Healthy Food Partnership [153]. The nutrient criteria within the proposed reformulation guideline apply to individual food categories allowing targets to be set in a format that is likely easier to understand and implement. These proposed targets will also apply nationally, eliminating any variability in criteria between states and territories, and revealing a potential way forwards for the institutional policies. Developing national institutional nutrition policies would require strong federal government leadership, supported by the state and territory governments, as the roles and responsibilities of the states and territories, and differences in health and education systems between jurisdictions [14], would require implementation to remain at the jurisdictional level.

This is the first study to investigate Australian state and territory nutrition policies across a variety of institutions. It focussed on government-led policies, as governments should be setting an example for creating healthy eating environments in publicly-funded institutions [7, 8]. The study highlighted discrepancies between jurisdictions regarding policy strength and comprehensiveness in identifying: policy gaps and critical areas for future work within schools, health facilities and workplaces, variations in the stringency of nutrition standards and nutrient criteria, and unique settings where policies could be implemented across all jurisdictions. Addressing these policy gaps and variations and expanding nutrition policies to cover a wider range of public settings would increase policy strength and comprehensiveness and likely impact on dietary intake and health. However, this study did not capture private-sector policies, or policies at local or federal level. The policies assessed were only relevant to free-living individuals, and did not capture policies relating to institutionalised individuals, such as hospital in-patients or prisoners (e.g. [155]). Policies were obtained through a manual search of state/territory health and education sector websites, supplemented by information from the Food Policy Index Australia website, and it is possible that some policies were missed. Lastly, this study only investigated differences in policy design, and did not assess the extent of implementation, barriers and enablers to implementation, or the impact of the policies on foods available, dietary intake or health 
outcomes. To date, much of the evaluative research has been led by the state/territory governments and data has been self-reported by institutions (e.g. [156, 157]) with minimal independent analyses or peer-review literature [20, 27]. High levels of non-compliance have also been previously highlighted [20,28], suggesting policies are not achieving their desired impact. As such there is a need for rigorous independent evaluation of these policies to determine the current status of policy implementation, identify strategies to improve policy uptake and compliance and determine policy impact on dietary intake and health outcomes.

\section{Conclusions}

Almost all states/territories in Australia have developed their own nutrition policies for schools, workplaces and health facilities, though policies vary between jurisdictions and institution types. This study highlights gaps in existing policies, such as a lack of monitoring and evaluation tools and timelines, which may be a barrier to implementation and prevent the policies having their intended impact. Rigorous independent evaluation of these policies is needed to identify strategies to improve uptake and compliance.

\section{Abbreviations}

ACT: Australian Capital Territory; NSW: New South Wales; NT: Northern Territory; QLD: Queensland; SA: South Australia; TAS: Tasmania; VIC: Victoria; WA: Western Australia; TL: Traffic light; HSR: Health Star Rating

\section{Acknowledgements}

Nil

\section{Authors' contributions}

ER collected the data and wrote the first draft of the manuscript with contributions from AH, CNM and JW. All authors read and approved the final manuscript.

\section{Funding}

ER is supported by a University of New South Wales University Postgraduate Award (UPA) (\#00889665) and George Institute Top-Up Scholarship.

$\mathrm{AH}$ is a researcher within the National Health and Medical Research Council (NHMRC) Centre for Research Excellence (CRE) on food policy interventions to reduce salt (\#1117300).

GS is supported by a Heart Foundation Future Leader Fellowship (\#102035) from the National Heart Foundation of Australia. He is also a researcher within the NHMRC CRE on food policy interventions to reduce salt (\#1117300) and on Food Retail Environments for Health (RE-FRESH) (\#1152968) (Australia). He has also received other funding from the NHMRC, Australian Research Council (ARC), Canadian Institutes of Health Research (CIHR) and the World Health Organization (WHO).

LW is supported by a NHMRC Career Development Fellowship (\#1128348) and Heart Foundation Future Leader Fellowship (\#101175) and is a Hunter New England Clinical Research Fellow.

JWu is supported by a University of New South Wales Scientia Fellowship and is a researcher in a NHMRC CRE on food policy interventions to reduce salt (\#1117300).

CNM receives funding from Health Research Council of New Zealand programme grants (18/672 and 16/443), the Healthier Lives He Oranga Hauora National Science Challenge, NHMRC CRE on food policy interventions to reduce salt (\#1117300), and NHMRC CRE on food retail environments for health (\#1152968).

JW receives funding from World Health Organization; VicHealth (\# 20122); National Heart Foundation Future Leaders Fellowship II (\#102039); NHMRC
CRE on food policy interventions to reduce salt (\#1117300) NHMRC project grants (\#1052555 and \#1111457).

\section{Availability of data and materials}

The datasets generated and analysed during the current study are publicly available from health and education department websites for each Australian state and territory government, from the Food Policy Index Australia website or other stakeholder websites, as referenced in the text.

\section{Ethics approval and consent to participate}

This study was approved by the University of New South Wales Human Research Ethics Advisory Panel (HC190242).

\section{Consent for publication}

Not applicable.

\section{Competing interests}

Nil to declare.

\section{Author details}

${ }^{1}$ The George Institute for Global Health, The University of New South Wales, Sydney, NSW 2052, Australia. ²Deakin University, Melbourne, Victoria 3125, Australia. ${ }^{3}$ University of Newcastle, Callaghan, NSW 2308, Australia. ${ }^{4}$ Victorian Health Promotion Foundation, 15-31 Pelham Street, Melbourne, Victoria 3053, Australia. ${ }^{5}$ The University of Auckland, Auckland 1142, New Zealand.

Received: 28 June 2019 Accepted: 22 June 2020

Published online: 30 June 2020

\section{References}

1. Institute for Health Metrics and Evaluation (IHME). GBD compare data visualization. 2017; https://vizhub.healthdata.org/gbd-compare/. Accessed 21 Apr 2020

2. Commonwealth of Australia. Healthy food partnership. 2016; http://www. health.gov.au/internet/main/publishing.nsf/content/healthy-foodpartnership. Accessed 21 Apr 2020.

3. Commonwealth of Australia. Food and health dialogue. 2015; http.//www.health. gov.au/internet/main/publishing.nsf/Content/fhd. Accessed 21 Apr 2020.

4. The Heart Foundation. Unpack the salt. 2019; https://unpackthesalt.com.au/. Accessed 21 Apr 2020.

5. Commonwealth of Australia. Health star rating system. 2018; http:// healthstarrating.gov.au/internet/healthstarrating/publishing.nsf/content/ home. Accessed 21 Apr 2020.

6. Australian Government Department of Health. 2010 National healthy school canteens guidelines for healthy foods and drinks supplied in school canteens 2014; http://www.health.gov.au/internet/main/publishing.nsf/ Content/5FFB6A30ECEE9321CA257BF0001DAB17/\$File/Canteen\%2 Oguidelines.pdf. Accessed 22 Feb 2019.

7. World Health Organization. SHAKE the salt habit: the SHAKE technical package for salt reduction. 2016; http://apps.who.int/iris/bitstream/10665/25 0135/1/9789241511346-eng.pdf. Accessed 21 Apr 2020.

8. World Health Organization. Creating an enabling environment for population-based salt reduction strategies. 2010; https:/apps.who.int/iris/bitstream/handle/10665/444 74/9789241500777_eng.pdf?sequence=1. Accessed 21 Apr 2020.

9. World Health Organization. Introduction: The right to health at work. Global strategy on occupational health for all: The way to health at work 1994; https://www.who.int/occupational_health/publications/globstrategy/en/ index $2 \mathrm{html}$. Accessed 21 Apr 2020

10. Organisation for Economic Co-operation and development (OECD). How long do students spend in the classroom? The school environment 2014; https://www.oecd-library.org/docserver/eag_highlights-2014-23-en. pdf?expires $=1550793520 \& i d=i d \& a c c n a m e=$ guest\&checksum=BA58DA3C933 07027947BC0C142D38D2F. Accessed 21 Apr 2020

11. Niebylski M, Lu T, Campbell N, et al. Healthy food procurement policies and their impact. Int J Environ Res Public Health. 2014;11(3):2608-27.

12. Trieu K, Neal B, Hawkes C, et al. Salt reduction initiatives around the world - A systematic review of Progress towards the global target. PLoS One. 2015;10(7): e0130247.

13. L'Abbé M, Schermel A, Minaker $L$, et al. Monitoring foods and beverages provided and sold in public sector settings. Obesity Rev. 2013;14:96-107. 
14. Parliamentary Education Office (PEO). Three levels of law-making fact sheet. 2018; https://www.peo.gov.au/uploads/peo/docs/fact-sheets/three_levels_ law-making.pdf. Accessed 21 Apr 2020.

15. ACT Government Education Directorate. ACT public school food and drink policy. 2017; https://www.education.act.gov.au/publications_and_policies/ School-and-Corporate-Policies/school-administration-and-management/ food-and-drink/act-public-school-food-and-drink-policy/act-public-schoolfood-and-drink-policy. Accessed 21 Apr 2020.

16. ACT Health. Healthy food and drink choices policy. 2013; http://www.health act.gov.au/sites/default/files/2019-02/Healthy\%20Food\%20and\%20Drink\%2 OChoices.docx. Accessed 21 Apr 2020.

17. Lucas P, Patterson E, Sacks G, Billich N, Evans C. Preschool and school meal policies: an overview of what we know about regulation, implementation, and impact on diet in the UK, Sweden, and Australia. Nutrients. 2017:9(7):736.

18. Lawlis T, Knox M, Jamieson M. School canteens: A systematic review of the policy, perceptions and use from an Australian perspective. Nutr Diet. 2016; 73(4):389-98.

19. Woods J, Bressan A, Langelaan C, Mallon A, Palermo C. Australian school canteens: menu guideline adherence or avoidance? Health Promotion J Australia. 2014;25(2):110-5.

20. Wu JH, Berg J, Neeson M. Overview of development and implementation of school canteen nutrition guidelines in Australia. J Home Econ Institute Australia. 2016;23(1):2.

21. Nathan N, Yoong SL, Sutherland R, et al. Effectiveness of a multicomponent intervention to enhance implementation of a healthy canteen policy in Australian primary schools: a randomised controlled trial. Int JBehav Nutr Phys Activity. 2016;13(1):106.

22. Wolfenden L, Nathan $\mathrm{N}$, Janssen L, et al. Multi-strategic intervention to enhance implementation of healthy canteen policy: a randomised controlled trial. Implementation Science. 2017;12(1):6.

23. Yoong SL, Nathan N, Wolfenden L, et al. CAFÉ: a multicomponent audit and feedback intervention to improve implementation of healthy food policy in primary school canteens: a randomised controlled trial. Int J Behav Nutr Phys Activity. 2016;13(1):126.

24. NSW Ministry of Health. NSW healthy school canteen strategy food and drink criteria. 2017; https://healthyschoolcanteens.nsw.gov.au/canteenmanagers/the-food-and-drink-criteria. Accessed 21 Apr 2020.

25. Northern Territory Government Department of Education. School nutrition and healthy eating policy. 2017; https://education.nt.gov.au/_data/assets/ pdf_file/0009/257805/school-nutrition-and-healthy-eating-policy.pdf. Accessed 21 Apr 2020.

26. Government of Western Australia Department of Education. Healthy food and drink in public schools policy and procedures; 2018. p. 15. Available at: http:// det.wa.edu.au/policies/detcms/policy-planning-and-accountability/policiesframework/policies/healthy-food-and-drink-policy.en?cat-id=3457102.

27. Tsai C, Svensen E, Flood V, et al. Healthiness of food and beverages for sale at two public hospitals in New South Wales, Australia. Nutrients. 2018;10(2):216.

28. Bell C, Pond N, Davies L, Francis JL, Campbell E, Wiggers J. Healthier choices in an Australian health service: a pre-post audit of an intervention to improve the nutritional value of foods and drinks in vending machines and food outlets. BMC Health Serv Res. 2013;13(1):492.

29. Deakin University. Food policy index Australia. 2019; https://www. foodpolicyindex.org.au/. Accessed 21 Apr 2020.

30. ACT Health. Healthy food and drink choices factsheet. 2015; https://www. health.act.gov.au/sites/default/files/2018-09/Healthy_Choices_Background_ FINAL.pdf. Accessed 21 Apr 2020.

31. ACT Health. Looking for fundraising alternatives? 2016; http://www.actnss. org/assets/Healthy-Fundraising-200916.pdf. Accessed 21 Apr 2020.

32. ACT Health. Think outside the snag. 2017; http://www.actnss.org/assets/ Documents/Think-outside-the-snag-2017-reduced.pdf. Accessed 21 Apr 2020.

33. NSW Ministry of Health. Healthy food and drink in NSW health facilities for staff and visitors framework. 2017; https://www.health.nsw.gov.au/heal/ Publications/hfd-framework.pdf. Accessed 21 Apr 2020.

34. NSW Ministry of Health. Healthy food and drink in NSW health facilities for staff and visitors framework. Healthy choices in health facilities toolkit. 2017; https://www.health.nsw.gov.au/heal/Publications/hfd-toolkit.pdf. Accessed 21 Apr 2020.

35. Northern Territory Government. Healthy choices made easy - healthy food and drink options for staff, volunteer and visitors, in NT health facilities. 2017; https://digitallibrary.health.nt.gov.au/prodjspui/bitstream/10137/904/3/
Healthy\%20Food\%20and\%20Drink\%200ptions\%20for\%20Staff\%2C\%2 OVolunteers\%20and\%20Visitors\%20in\%20NT\%20Health\%20Facilities\%2 OPolicy.pdf. Accessed 21 Apr 2020.

36. Northern Territory Government. Healthy choices made easy: quick quide to the policy 2017; https://digitallibrary.health.nt.gov.au/prodjspui/bitstream/1 0137/864/3/Quick\%20Guide\%20to\%20the\%20Healthy\%20Food\%20and\%2 0Drink\%200ptions\%20in\%20NT\%20Health\%20Facilities\%20Policy.pdf. Accessed 21 Apr 2020.

37. Northern Territory Government. Healthy choices made easy - catering guide for meetings, functions, events and health education activities. 2017; https:// digitallibrary.health.nt.gov.au/prodjspui/bitstream/10137/900/3/Catering\%2 0Guide\%20for\%20Meetings\%20Functions\%20Events\%20and\%20Health\%2 OEducation\%20Activities\%20v10.pdf. Accessed 21 Apr 2020

38. Northern Territory Government. Healthy choices made easy - healthy food and drinks. 2017; https://digitallibrary.health.nt.gov.au/prodjspui/bitstream/1 0137/901/3/Healthy\%20Choices\%20Made\%20Easy\%20Foods\%20and\%2 0Drinks\%20Guide.pdf. Accessed 21 Apr 2020.

39. Northern Territory Government. Healthy choices made easy - healthy fundraising guide. 2017; https://digitallibrary.health.nt.gov.au/prodjspui/ bitstream/10137/857/3/Healthy\%20Choices\%20Made\%20Easy\%2 OFundraising\%20Guide.pdf. Accessed 21 Apr 2020.

40. Northern Territory Government. Healthy choices made easy - vending machines RDH guideline. 2017; https://digitallibrary.health.nt.gov.au/ prodjspui/bitstream/10137/714/1/Nending\%20Machines\%20RDH\%2 OGuideline\%20Updated\%200ct\%202016.docx. Accessed 21 Apr 2020.

41. Queensland Health. A better choice. Healthy food and drink supply strategy for Queensland health facilities. 2007; https://www.health.qld.gov.au/_data/ assets/pdf_file/0025/437272/32511.pdf. Accessed 25 Feb 2019.

42. Queensland Health. A better choice. Catering guidelines for meetings and functions. 2007; https://www.health.qld.gov.au/__data/assets/pdf_file/001 9/443053/catering-guidelines-functions.pdf. Accessed 25 Feb 2019.

43. Queensland Health. A better choice. toolkit. 2008; https://www.health.qld.gov. au/_data/assets/pdf_file/0033/438729/toolkit.pdf. Accessed 25 Feb 2019.

44. Queensland Health. Healthier Drinks at Healthcare Facilities. Best Practice Guide. Unknown; https://www.health.qld.gov.au/_data/assets/pdf_file/ 0023/656024/best-practice-healthier-drinks.pdf. Accessed 25 Feb 2019.

45. Queensland Health. Healthy Food and Drink Supply (A Better Choice). 2015; https://www.health.qld.gov.au/_data/assets/pdf_file/0026/147473/qhgdl-344.pdf. Accessed 25 Feb 2019.

46. SA Health. Getting ready for healthier choices fact sheet 1. 2009; https:// www.sahealth.sa.gov.au/wps/wcm/connect/2137e4804fb84efebbd0bb5cbc1 eale9/Fact+Sheet+1.pdf?MOD=AJPERES\&CACHEID=ROOTWORKSPACE-213 7e4804fb84efebbd0bb5cbc1ea1e9-mwMR3aX. Accessed 21 Apr 2020.

47. SA Health. Recommended supply of food and drinks fact sheet 22009 ; https://www.sahealth.sa.gov.au/wps/wcm/connect/873356004fb84e83 bb77bb5cbclea1e9/Fact+Sheet+2.pdf?MOD=AJPERES\&CACHEID= ROOTWORKSPACE-873356004fb84e83bb77bb5cbc1ea1e9-mwN5g2j. Accessed 21 Apr 2020.

48. SA Health. Summary of nutrient criteria fact sheet 3. 2009; https:// www.sahealth.sa.gov.au/wps/wcm/connect/78a55a004fb84e03bb3fbb5 cbclea1e9/Fact+Sheet+3.pdf?MOD=AJPERES\&CACHEID= ROOTWORKSPACE-78a55a004fb84e03bb3fbb5cbc1ea1e9-mwNaTt5. Accessed 21 Apr 2020.

49. SA Health. Healthy catering ideas for meetings, functions and events fact sheet 4 2009; https://www.sahealth.sa.gov.au/wps/wcm/connect/0a14bd804 fb84d6bbb00bb5cbclea1e9/Fact+sheet+4.pdf?MOD=AJPERES\&CACHEID= ROOTWORKSPACE-0a14bd804fb84d6bbb00bb5cbc1ea1e9-mwMTVuL. Accessed 21 Apr 2020.

50. SA Health. Fundraising ideas fact sheet 5. 2009; https://www.sahealth.sa.gov. au/wps/wcm/connect/3f36e8004fb84c65baa8ba5cbc1ea1e9/fact+sheet+5. pdf?MOD=AJPERES\&CACHEID=ROOTWORKSPACE-3f36e8004fb84c65 baa8ba5cbc1ea1e9-mwMQvN. Accessed 21 Apr 2020.

51. SA Health. Healthy food and drink choices for staff and visitors in SA health facilities policy. 2011; https://www.sahealth.sa.gov.au/wps/wcm/connect/5d6 8ac80465eee648649fe2e504170d4/HealthyFoodDrinkChoices-PHCS-HPB-11 03.pdf?MOD=AJPERES\&CACHEID=ROOTWORKSPACE-5d68ac80465eee64864 9fe2e504170d4-m2DDq87. Accessed 21 Apr 2020.

52. SA Health. Healthy food and drink choices for staff and visitors in SA health facilities policy. Quick guide. 2011; https://www.sahealth.sa.gov.au/wps/ wcm/connect/2ab3a4804ef6d509b8a1bb6a7ac0d6e4/Quick+Guide+-+ Healthy+Food+and+Drink+Choices+for+Staff.pdf?MOD= 
AJPERES\&CACHEID=ROOTWORKSPACE-2ab3a4804ef6d509b8a 1 bb6a7ac0d 6 e4-mwMT-yk. Accessed 21 Apr 2020.

53. SA Health. Healthy food and drink choices for staff and visitors in SA health facilities food and drinks guide. 2011; https://www.sahealth.sa.gov.au/wps/ wcm/connect/4fcae9804fb846ccb8a8ba5cbcleale9/Food+and+Drinks+ Guide.pdf?MOD=AJPERES\&CACHEID=ROOTWORKSPACE-4fcae9804fb846 ccb8a8ba5cbclea1e9-mwMT.di. Accessed 21 Apr 2020.

54. Department of Health and Human Services State of Victoria. Healthy choices: food and drink classification guide. A system for classifying foods and drinks. 2016; https://www2.health.vic.gov.au/Api/downloadmedia/\% 7B934172DF-6E0E-48C7-91F8-53E25EB764F7\%7D. Accessed 21 Apr 2020.

55. Department of Health and Human Services State of Victoria. Healthy choices: policy guidelines for hospitals and health services. 2016; https:// www2.health.vic.gov.au/Api/downloadmedia/\%7B087EEAAD-7C83-49E0A562-D956CCC5ED0C\%7D. Accessed 21 Apr 2020.

56. Government of Western Australia Department of Health. Healthy options WA. Food and nutrition policy for WA health services and facilities. 2015; https://ww2.health.wa.gov.au/ /media/Files/Corporate/Policy\%2 OFrameworks/Public\%20Health/Policy/Healthy\%200ptions\%20WA\%2 OFood\%20and\%20Nutrition\%20Policy/OD627-Healthy-Options-WA-Foodand-Nutrition-Policy-for-WA-Health.pdf. Accessed 21 Apr 2020.

57. Government of Western Australia Department of Health. Healthy options WA. Tips to promote and sell green items. 2016; https://ww2.health.wa.gov. $\mathrm{au} /$ /media/Files/Corporate/general\%20documents/HealthyOptions/PDF/ promoting-green.pdf. Accessed 21 Apr 2020

58. Government of Western Australia Department of Health. Healthy options WA. Making healthy food choices easier. Implementation guide. 2017; https://ww2.health.wa.gov.au/ /media/Files/Corporate/general\%2 Odocuments/HealthyOptions/170830_HO_Implementation_Guide_2017.pdf. Accessed 21 Apr 2020

59. Government of Western Australia Department of Health. Healthy options WA. Commonly supplied food and drink guide. 2017; https://ww2.health.wa. gov.au/ /media/Files/Corporate/general\%20documents/HealthyOptions/ commonly-supplied-food-and-drink-guide.pdf. Accessed 21 Apr 2020.

60. Government of Western Australia Department of Health. Healthy options WA. Classification of green, amber and red food and drink. 2017; https://ww2. health.wa.gov.au/ /media/Files/Corporate/general\%20documents/ HealthyOptions/PDF/160420_Colour_Classification.pdf. Accessed 21 Apr 2020

61. Government of Western Australia Department of Health. Healthy Options WA. Self-assessment Tool. Unknown; https://ww2.health.wa.gov.au/ /media/ Files/Corporate/general\%20documents/HealthyOptions/PDF/160421_Selfassessment-tool.pdf. Accessed 21 Apr 2020.

62. ACT Government Education Directorate. Amber food and drink items fact sheet. 2015; https:/www.education.act.gov.au/publications_and_policies/ School-and-Corporate-Policies/school-administration-and-management/ food-and-drink?a=691773. Accessed 21 Apr 2020.

63. ACT Government Education Directorate. Green food and drink items fact sheet. 2015; https://www.education.act.gov.au/publications_and_policies/ School-and-Corporate-Policies/school-administration-and-management/ food-and-drink?a=691717. Accessed 21 Apr 2020.

64. ACT Government Education Directorate. ACT public school food and drink policy 2015 fact sheet. 2018; https://www.education.act.gov.au/ publications_and_policies/School-and-Corporate-Policies/schooladministration-and-management/food-and-drink?a=692297. Accessed 21 Apr 2020

65. ACT Government Education Directorate. Red food and drink items fact sheet. 2018; https://www.education.act.gov.au/publications_and_policies/ School-and-Corporate-Policies/school-administration-and-management/ food-and-drink?a=691733. Accessed 21 Apr 2020.

66. ACT Government Education Directorate. Fresh tastes: healthy food at school fact sheet. 2018; https://www.education.act.gov.au/publications_and_ policies/School-and-Corporate-Policies/school-administration-andmanagement/food-and-drink?a=691713. Accessed 21 Apr 2020.

67. ACT Government Education Directorate. Sugary drinks are phased out fact sheet. 2018; https://www.education.act.gov.au/publications_and_policies/ School-and-Corporate-Policies/school-administration-and-management/ food-and-drink?a=691749. Accessed 21 Apr 2020.

68. ACT Government Education Directorate. Healthy living - healthy fundraising options factsheet. 2018; https:/www.education.act.gov.au/publications_ and_policies/School-and-Corporate-Policies/school-administration-andmanagement/food-and-drink?a=1078407. Accessed 21 Apr 2020.
69. ACT Government Fresh Tastes. Fresh tastes strategies to support the creation of a healthy food and drink culture in ACT schools. 2014; https:// goodhabitsforlife.act.gov.au/kids-at-play/sites/goodhabitsforlife.act.gov.au. fresh-tastes/files/Fresh\%20Tastes\%20strategy_0.pdf. Accessed 21 Apr 2020.

70. ACT Government Fresh Tastes. Go for green using the traffic light system healthy food and drinks for children. 2017; https:/goodhabitsforlife.act.gov. au/fresh-tastes/sites/goodhabitsforlife.act.gov.au.fresh-tastes/files/Go_for_ Green.pdf. Accessed 21 Apr 2020.

71. NSW Ministry of Health. NSW healthy school canteen strategy selfassessment tool. 2017; https://healthyschoolcanteens.nsw.gov.au/media/ resource-centre/guides-and-tools/Self-Assesment-Tool-for-School-Planning. pdf. Accessed 21 Apr 2020.

72. NSW Ministry of Health. NSW healthy school canteen strategy menu check application form. 2017; https://healthyschoolcanteens.nsw.gov.au/media/ resource-centre/guides-and-tools/Menu-Check-application.pdf. Accessed 21 Apr 2020.

73. NSW Ministry of Health. NSW healthy school canteen strategy recipe checklist. 2017; https://healthyschoolcanteens.nsw.gov.au/media/resourcecentre/guides-and-tools/Recipe-Checklist.pdf. Accessed 21 Apr 2020.

74. NSW Ministry of Health. NSW healthy school canteen strategy ready, set, choose your action list. 2017; https://healthyschoolcanteens.nsw.gov.au/ media/documents/Ready-Set-Choose-action-list.pdf. Accessed 21 Apr 2020.

75. NSW Department of Education. Nutrition in schools policy. 2017; https:// education.nsw.gov.au/policy-library/policies/nutrition-in-schools-policy. Accessed 21 Apr 2020.

76. Northern Territory Government Department of Education. School nutrition and healthy eating guidelines. 2017; https://education.nt.gov.au/_data/ assets/pdf_file/0008/257804/school-nutrition-healthy-eating-guidelines.pdf. Accessed 21 Apr 2020.

77. Northern Territory Government Department of Education. Creating a healthier menu. 2017; https://education.nt.gov.au/_data/assets/pdf_file/ 0003/257826/creating-a-healthier-menu.pdf. Accessed 21 Apr 2020.

78. Northern Territory Government Department of Education. How to decide if a food or drink is suitable for school. 2017; https://education.nt.gov.au/_ data/assets/pdf_file/0005/462056/ground-rules-for-assessing-meals.pdf. Accessed 21 Apr 2020.

79. Northern Territory Government Department of Education. Healthy alternatives to sausage sizzles. 2017; https://education.nt.gov.au/_data/ assets/pdf_file/0010/257824/healthy-alternative-to-sausage-sizzles.pdf. Accessed 21 Apr 2020.

80. Northern Territory Government Department of Education. Ideas for fundraising. 2017; https://education.nt.gov.au/_data/assets/pdf_file/0009/25 7823/ideas-for-fundraising.pdf. Accessed 21 Apr 2020.

81. Northern Territory Government Department of Education. Healthy food based fundraising. 2017; https://education.nt.gov.au/_data/assets/pdf_file/ 0008/257822/healthy-food-based-fundraising.pdf. Accessed 21 Apr 2020.

82. Northern Territory Government Department of Education. Recipe checklist. 2017; https://education.nt.gov.au/_data/assets/pdf_file/0011/257825/ recipe-checklist.pdf. Accessed 21 Apr 2020.

83. Northern Territory Government Department of Education. Sporting events, fundraising ideas and non-food rewards. 2017; https://education.nt.gov. au/_data/assets/pdf_file/0007/257821/sporting-events-fundraising-ideasand-non-food-rewards.pdf. Accessed 21 Apr 2020.

84. Northern Territory Government Department of Education. School camp ideas and BBQ catering. 2017; https://education.nt.gov.au/_data/assets/ pdf_file/0006/257820/school-camp-ideas-and-barbecue-catering.pdf. Accessed 21 Apr 2020.

85. Queensland Health and Education Queensland. Smart choices. Healthy food and drink supply strategy for Queensland schools. 2016; https:/education.qld. gov.au/student/Documents/smart-choices-strategy.pdf. Accessed 21 Apr 2020.

86. Queensland Health and Education Queensland. Smart Choices @ School Events. 2016; https://education.qld.gov.au/student/Documents/smartchoices-school-events.pdf. Accessed 21 Apr 2020.

87. Government of South Australia Department for Education and Child Development. Eat well SA schools and preschools healthy eating guidelines. 2004; https://www.education.sa.gov.au/sites/g/files/net691/f/healthy_eating_ guidelines.pdf. Accessed 21 Apr 2020.

88. Government of South Australia Department for Education and Child Development. Right bite, healthy food and drink supply strategy for South Australian schools and preschools. 2008; https:/www.education.sa.gov.au/ sites/g/files/net691/f/right_bite_manual_colour.pdf. Accessed 21 Apr 2020. 
89. Government of South Australia Department for Education and Child Development. Right bite, easy guide to healthy food and drink supply for South Australian schools and preschools. 2015; https://www.education.sa. gov.au/sites/g/files/net691/f/easy_guide_to_healthy_food_and_drink_ supply_in_sa_schools_and_preschools.pdf. Accessed 21 Apr 2020.

90. Government of South Australia Department for Education and Child Development. Right Bite, Food Supply Checklist. Unknown; https://www. education.sa.gov.au/sites/g/files/net691/f/right_bite_food_supply_checklist. pdf. Accessed 21 Apr 2020.

91. Tasmanian School Canteen Association Inc. School canteen handbook. A whole school approach to healthy eating. 2014; http://tascanteenassn.org. au/wp-content/uploads/2014/08/Accreditation-Folder-FULL.pdf. Accessed 21 Apr 2020.

92. Cancer Council Victoria. Healthy schools. 2018; https://wuw.achievementprogram. health.vic.gov.au/healthy-places/schools. Accessed 21 Apr 2020.

93. State of Victoria Department of Education and Early Childhood Development. Healthy canteen kit. School canteens and other school food services policy. 2012; https:/www.education.vic.gov.au/Documents/school/principals/ management/gfylpolicy.pdf. Accessed 21 Apr 2020.

94. State of Victoria Department of Education and Early Childhood Development. Healthy canteen kit. School confectionary guidelines. 2012; https://www.education.vic.gov.au/Documents/school/principals/ management/impcanteenconfp.pdf. Accessed 21 Apr 2020.

95. State of Victoria Department of Education and Early Childhood Development. Healthy Canteen Kit. Food Planner. Unknown; https://www education.vic.gov.au/Documents/school/principals/management/gfylplan. pdf. Accessed 21 Apr 2020

96. Government of Western Australia Department of Education. FOCis nutrient criteria; 2018. p. 15. Available at: http://det.wa.edu.au/redirect/?oid=com. arsdigita.cms.contenttypes. FileStorageltem-id-11238770\&stream_asset=true. Accessed 21 Apr 2020

97. Government of Western Australia Department of Education. How to market healthy foods. 2018; http://det.wa.edu.au/redirect/?oid=com.arsdigita.cms. contenttypes. FileStorageltem-id-11238840\&stream_asset=true. Accessed 21 Apr 2020.

98. Government of Western Australia Department of Education. Healthy schools: events and fundraising by schools and parants and citizens' associations fundraising; 2018. p. 15. Available at: http://det.wa.edu.au/ redirect/?oid=com.arsdigita.cms.contenttypes. FileStorageltem-id11238612\&stream_asset=true. Accessed 21 Apr 2020.

99. Federation of Canteens in Schools (FOCiS). FOCiS product registration program - food industry handbook. 2017; https://www.focis.com.au/wpcontent/uploads/2017/07/FOCiS-Food-Industry-Handbook-July-2017.pdf. Accessed 21 Apr 2020

100. ACT Government Healthier Work. Guide to promoting health and wellbeing in the workplace. 2016; http://www.healthierwork.act.gov.au/wp-content/ uploads/2015/01/Guide-to-Promoting-Health-and-Wellbeing-in-theWorkplace-2016.pdf. Accessed 21 Apr 2020.

101. ACT Government Healthier Work. ACT healthy workplaces audit tool. 2012 http://www.healthierwork.act.gov.au/wp-content/uploads/2015/01/HW_ Audit_Tool.pdf. Accessed 21 Apr 2020.

102. ACT Government Healthier Work. Health and wellbeing policy example. 2012; http://www.healthierwork.act.gov.au/wp-content/uploads/2015/01/ HW_Policy_Example.pdf. Accessed 21 Apr 2020.

103. ACT Government Healthier Work. Health and wellbeing action plan example. 2012; http://www.healthierwork.act.gov.au/wp-content/uploads/2 015/01/HW_Action_Plan_template.pdf. Accessed 21 Apr 2020.

104. ACT Government Healthier Work. Health and wellbeing policy template. 2012; http://www.healthierwork.act.gov.au/wp-content/uploads/2015/01/ Health_and_Wellbeing_Policy_Template.doc. Accessed 21 Apr 2020.

105. ACT Government Healthier Work. Health and wellbeing action plan template. 2012; http://www.healthierwork.act.gov.au/wp-content/uploads/2 015/01/ACTIONPLAN_TEMPLATE.docx. Accessed 21 Apr 2020.

106. ACT Government Healthier Work. Workplace health evaluation overview. 2012; http://www.healthierwork.act.gov.au/wp-content/uploads/2015/01/ HW_Evaluation_Overview.pdf. Accessed 21 Apr 2020.

107. ACT Government Healthier Work. Health and wellbeing action plan example. 2012; http://www.healthierwork.act.gov.au/wp-content/uploads/2 015/01/HW_Action_Plan_template.pdf. Accessed 22 Feb 2019.

108. NSW Government Get Healthy at Work. Healthier food \& drinks guide. 2017; https://gethealthyatwork.com.au/ /media/Files/Resources/Healthy\%2
Oeating/NSWMH0132_Healthier\%20Food\%20and\%20Drinks\%20Guide.ashx. Accessed 21 Apr 2020.

109. NSW Government Get Healthy at Work. Healthier food \& drink facilities. 2017; http://www.gethealthyatwork.com.au/ /media/Files/Resources/ Healthy\%20eating/NSWMH0130_Healthier\%20Food\%20and\%20Drink\%2 OFacilities.ashx. Accessed 21 Apr 2020

110. NSW Government Get Healthy at Work. Workplace guide to healthier vending machines. 2017; http://www.gethealthyatwork.com.au/ /media/ Files/Resources/Healthy\%20eating/NSWH0129_Healthier\%20Vending\%2 OMachines.ashx. Accessed 21 Apr 2020.

111. NSW Government Get Healthy at Work. Healthier fundraising: a guide. 2017; http://www.gethealthyatwork.com.au/ /media/Files/Resources/Healthy\%2 Oeating/NSWMH0131_Healthier\%20Fundraising.ashx. Accessed 21 Apr 2020.

112. Northern Territory Government. The healthy workplace toolkit. Your simple guide to workplace health and wellbeing. 2017; https:// digitallibrary.health.nt.gov.au/prodjspui/bitstream/10137/2725/1/ Healthy\%20Workplace\%20Toolkit.pdf. Accessed 21 Apr 2020

113. Queensland Government Healthier Happier Workplaces. Step 1. management commitment. 2019; https://workplaces.healthier.qld.gov.au/ getting-started/step-one-management-commitment/. Accessed 21 Apr 2020.

114. Queensland Government Healthier Happier Workplaces. Step 2. wellness planning. 2019; https://workplaces.healthier.qld.gov.au/getting-started/steptwo-wellness-planning/. Accessed 21 Apr 2020.

115. Queensland Government Healthier Happier Workplaces. Step 3. Needs Assessment. 2019; https://workplaces.healthier.qld.gov.au/getting-started/ step-three-needs-assessment/. Accessed 21 Apr 2020.

116. Queensland Government Healthier Happier Workplaces. Step 4. Action Plan. 2019; https://workplaces.healthier.qld.gov.au/getting-started/step-fouraction-plan/. Accessed 21 Apr 2020.

117. Queensland Government Healthier Happier Workplaces. Step 5. Evaluation. 2019; https://workplaces.healthier.qld.gov.au/getting-started/step-fiveevaluation/. Accessed 21 Apr 2020.

118. SA Health Healthy Workers - Healthy Futures Initiative. A workplace health and wellbeing toolkit: step by step guide to developing a successful workplace program. 2014; https://www.sahealth.sa.gov.au/ wps/wcm/connect/public+content/sa+health+internet/resources/ healthy+workers+-+healthy+futures+a+workplace+health+and+ wellbeing+toolkit. Accessed 21 Apr 2020.

119. WorkCover Tasmania. Healthy workplace nutrition guidelines. 2013; https://www.worksafe.tas.gov.au/__data/assets/pdf_file/0004/288184/ Healthy_workplace_nutrition_guidelines_fact_sheet.pdf. Accessed 21 Apr 2020

120. WorkSafe Tasmania, Public Health Services. Your simple guide to workplace health and wellbeing. 2013; https://www.worksafe.tas.gov.au/_data/assets/ pdf_file/0003/252390/Your-simple-guide-to-workplace-health-and-wellbeing. pdf. Accessed 21 Apr 2020.

121. WorkSafe Tasmania, Public Health Services. 06A Healthy Eating. 2013; https://www.worksafe.tas.gov.au/_data/assets/pdf_file/0010/288181/06A_ Healthy_eating.pdf. Accessed 21 Apr 2020.

122. Department of Health and Human Services State of Victoria. Healthy choices: healthy eating policy and catering guide for workplaces. 2016; https://www2.health.vic.gov.au/Api/downloadmedia/\%7BEB1CFE60-E816-4 C26-820E-4EFAA9D0983E\%7D. Accessed 21 Apr 2020.

123. Healthier Workplace WA. 3 Steps for a healthier workplace: plan, do, review. 2017; https://healthierworkplacewa.com.au/media/130392/plan-do-reviewinteractive-guide.pdf. Accessed 21 Apr 2020

124. Healthier Workplace WA. Gettng Started. 2017; https://healthierworkplacewa. com.au/take-action/get-started/develop-a-program/plan/getting-started/. Accessed 21 Apr 2020

125. Healthier Workplace WA. Implementing Your Program. 2017; https:// healthierworkplacewa.com.au/take-action/get-started/develop-aprogram/do/implementing-your-program/. Accessed 21 Apr 2020.

126. Healthier Workplace WA. Evaluating Your Program. 2017; https:// healthierworkplacewa.com.au/take-action/get-started/develop-a-program/ review/evaluating-your-program/. Accessed 21 Apr 2020.

127. Healthy Choices Healthy Futures. Healthier Fundraising: A Guide. 2019; http://hchf.com.au/system/resources/resource_assets/000/000/021/original/ 0398_HCHF_Fundraising_Guide.pdf?1396837401. Accessed 21 Apr 2020.

128. Healthy Choices Healthy Futures. Healthier Gifts \& Rewards: Alternatives to Wine \& Chocolate. 2019; http://hchf.com.au/system/resources/resource_ 
assets/000/000/022/original/0398_HCHF_Gifts_and_Rewards.pdf?139683 7336. Accessed 21 Apr 2020.

129. Healthy Choices Healthy Futures. Healthier Food and Drink Facilities. 2019; http://hchf.com.au/system/resources/resource_assets/000/000/015/original/ 0398_HCHF_Healthier_Facilities.pdf?1396837538. Accessed 21 Apr2020.

130. Healthy Choices Healthy Futures. Healthier Vending Machines: An Employer Guide. 2019; http://hchf.com.au/system/resources/resource_assets/000/000/ 032/original/HCHF_Healthier_Vending_Machines.pdf?1397094488. Accessed 21 Apr 2020

131. Healthy Choices Healthy Futures. Healthier Food and Drink Guide. 2019; http://hchf.com.au/system/resources/resource_assets/000/000/055/original/ Final_HFD_Guide_2016.pdf?1466653177. Accessed 21 Apr 2020.

132. Healthy Choices Healthy Futures. Healthier Catering: A Guide to Assist Workplaces. 2019; http://hchf.com.au/system/resources/resource_assets/000/ 000/007/original/0398_HCHF_Healthier_Catering.pdf?1396837711. Accessed 21 Apr 2020.

133. Department of Health and Human Services State of Victoria. Healthy Choices: Policy Guidelines for Parks. 2016; https://www2.health.vic.gov.au/ Api/downloadmedia/\%7BE4A9127E-868B-4628-A7EE-64A79A0AE982\%7D. Accessed 21 Apr 2020.

134. ACT Health. ACT Public Sector Healthy Food and Drink Choices Policy. 2016; http://www.cmd.act.gov.au/_data/assets/pdf_file/0003/905772/WHS-01-2 016-Healthy-Food-and-Drink-Choices-Policy.pdf. Accessed 21 Apr 2020

135. ACT Health. ACT Public Sector Healthy Food and Drink Choices Vending Machine Management. 2016; http://www.cmd.act.gov.au/_data/assets/pdf_ file/0012/667893/WHS-02-2014-Healthy-Food-and-Drink-Choices-VendingMachine-Management.pdf. Accessed 21 Apr 2020.

136. Workplace Health and Safety Queensland. Choose Well, Live Well. Guidelines for the Implementation of Nutrition Standards and Point of Choice Promotions in Australian Remote Area Camps. 2014; https://www. worksafe.qld.gov.au/_data/assets/pdf_file/0017/114920/choose-well-livewell-booklet.pdf. Accessed 21 Apr 2020.

137. Department of Health and Human Services State of Victoria. Healthy Choices: Policy Guidelines for Sport and Recreation Centres. 2016; https:// www2.health.vic.gov.au/Api/downloadmedia/\%7B7EF29993-EEC4-414FB942-ADF75729E734\%7D. Accessed 21 Apr 2020.

138. National Health and Medical Research Council. Australian dietary guidelines. 2013; www.nhmrc.gov.au/guidelines-publications/n55. Accessed 21 Apr 2020.

139. NSW Department of Health. Fresh Tastes @ School. NSW health school canteen strategy. 2006; https://web.archive.org/web/20110516043316/http:// www.schools.nsw.edu.au/media/downloads/schoolsweb/studentsupport/ studentwellbeing/schoolcanteen/cmpguide2.pdf. Accessed 21 Apr 2020.

140. Meyers DC, Durlak JA, Wandersman A. The quality implementation framework: a synthesis of critical steps in the implementation process. Am J Community Psychol. 2012;50(3-4):462-80.

141. Mozaffarian D, Angell SY, Lang T, Rivera JA. Role of government policy in nutrition-barriers to and opportunities for healthier eating. BMJ (Clinical research ed). 2018;361:k2426.

142. Ardzejewska K, Tadros R, Baxter D. A descriptive study on the barriers and facilitators to implementation of the NSW (Australia) Healthy school canteen strategy. Health Educ J. 2013;72(2):136-45.

143. Reilly K, Wyse R, Yoong S, Nathan N. Is the banning of unhealthy foods at school canteens associated with principal influence or school policy? Obes Res Clin Pract. 2014;8:85.

144. Rana L, Alvaro R. Applying a Health promoting schools approach to nutrition interventions in schools: key factors for success. Health Promot J Austr. 2010;21(2):106-13.

145. Reeve E, Thow AM, Bell C, et al. Implementation lessons for school food policies and marketing restrictions in the Philippines: a qualitative policy analysis. Glob Health. 2018;14(1):8.

146. Boelsen-Robinson T, Backholer K, Corben K, Blake MR, Palermo C, Peeters A. The effect of a change to healthy vending in a major Australian health service on sales of healthy and unhealthy food and beverages. Appetite. 2017;114:73.

147. Parliament of Canada. Canada's system of government. 2020; https://lop. parl.ca/about/parliament/education/ourcountryourparliament/html_booklet/ canada-s-system-government-e.html. Accessed 21 Apr 2020.

148. Department for Education. School food standards. A practical guide for schools their cooks and caterers. 2015; https://assets.publishing.service.gov. uk/government/uploads/system/uploads/attachment_data/file/847621/ School-Food-Standards-Guidance-FINAL-V3.pdf. Accessed 21 Apr 2020

149. New York City Health. New York City food standards meals/snacks purchased and served. 2018; https:/www1.nyc.gov/assets/doh/downloads/ pdf/cardio/cardio-meals-snacks-standards.pdf. Accessed 21 Apr 2020.

150. Woodward-Lopez G, Gosliner W, Samuels SE, Craypo L, Kao J, Crawford PB. Lessons learned from evaluations of California's statewide school nutrition standards. Am J Public Health. 2010;100(11):2137.

151. World Cancer Research Fund International. Offer healthy food and set standards in public institutions and other specific settings. 2018; https://www. wcrforg/sites/default/files/Offer-healthy-food.pdf. Accessed 21 Apr 2020.

152. Federation of Canteens in Schools (FOCiS). FOCiS nutrient criteria review 2016. 2016; https://www.focis.com.au/wp-content/uploads/2017/05/FOCiSNutrient-Criteria-review-Summary-Report.pdf. Accessed 21 Apr 2020.

153. Healthy Food Partnership. Healthy food partnership reformulation program: evidence informing the approach, draft targets and modelling outcomes. 2018; http://www.health.gov.au/internet/main/publishing.nsf/Content/ reformulation. Accessed 21 Apr 2020.

154. Sparks E, Farrand C, Santos J, et al. Sodium levels of processed meat in Australia: supermarket survey data from 2010 to 2017. Nutrients. 2018;10(11): 1686.

155. NSW Agency for Clinical Innovation. Nutrition standards for adult inpatients in NSW hospitals. 2011; https://www.aci.health.nsw.gov.au/_data/assets/ pdf_file/0004/160555/ACl_Adult_Nutrition_web.pdf. Accessed 21 Apr 2020.

156. Queensland Health and Department of Education and Training. Smart choices evaluation report healthy food and drink strategy for Queensland schools. 2009; https://education.qld.gov.au/student/Documents/evaluationreport.pdf. Accessed 24 Sept 2018.

157. SA Health. Healthy food and drink choices for staff and visitors in SA health facilities policy. Evaluation. Summary report of findings. 2012; https://www. sahealth.sa.gov.au/wps/wcm/connect/public+content/sa+health+internet/ resources/healthy+food+and+drink+choices+for+staff+and+visitors+in+sa+ health+facilities+policy+evaluation. Accessed 21 Apr 2020.

\section{Publisher's Note}

Springer Nature remains neutral with regard to jurisdictional claims in published maps and institutional affiliations.
Ready to submit your research? Choose BMC and benefit from:

- fast, convenient online submission

- thorough peer review by experienced researchers in your field

- rapid publication on acceptance

- support for research data, including large and complex data types

- gold Open Access which fosters wider collaboration and increased citations

- maximum visibility for your research: over $100 \mathrm{M}$ website views per year

At BMC, research is always in progress.

Learn more biomedcentral.com/submissions 